\title{
ON THE USE OF JENSEN'S INEQUALITY FOR MIMO CHANNEL CAPACITY ESTIMATION
}

\author{
S. Loyka, A. Kouki \\ Department of Electrical Engineering \\ Ecole de Technologie Superieure \\ 1100, Notre-Dame St. West \\ Montreal (Quebec), H3C 1K3, Canada \\ Email: sergey.loyka@ieee.org
}

\begin{abstract}
In this paper, we derive a universal upper bound on the MIMO channel capacity, which is not limited to a particular scenario, using the correlation matrix approach and the Jensen's inequality. This bound accounts for both transmit and receive branch correlation in such a way that the impact of these branches can be estimated separately, which simplifies the procedure substantially. Some simple analytical results, which quantify the impact of correlation on the MIMO capacity in an explicit way, are given. We show that correlation increase is equivalent to SNR decrease in some cases. Finally, we discuss the paradox of zero correlation and provide a statistical explanation for it. We demonstrate why zero mean correlation is not a guarantee of high capacity. Mean magnitude or RMS correlation should be used for the capacity estimation of degenerate MIMO channels.
\end{abstract}

\section{INTRODUCTION}

Multiple-input multiple-output (MIMO) communication architecture (also known as BLAST - Bell Labs Layered Space Time), which uses multiple antennas at both the transmitter and the receiver, has recently emerged as a new paradigm of extremely spectrum-efficient wireless communications in rich multipath environments [1-4]. Suffice it to say that unprecedented wireless spectral efficiencies, ranging from $20-40 \mathrm{bit} / \mathrm{s} / \mathrm{Hz}$, have been demonstrated in a laboratory environment [4], which are simply unattainable using traditional techniques. Even higher spectral efficiencies may be achieved in certain environments when the system design is optimal.

The central paradigm behind MIMO/BLAST is to use rather than to suppress multipath. Using a special signal processing algorithm at the receiver, the matrix wireless channel can be transformed into a virtual set of independent parallel channels in rich multipath environment. However, in real-life conditions the MIMO channel capacity may be limited due to several factors. One of the most important such factors is the correlation between sub-channels (i.e., links between one transmit and one receive antenna) of the matrix channel [5-7]. The MIMO capacity achieves its maximum for completely uncorrelated matrix channel. The correlation between individual receive and/or transmit branches results in capacity decrease. Several models have been used so far to study this phenomenon. Their application is typically limited to some specific scenarios.

In this paper, we derive the universal upper bound on the MIMO channel capacity, which is not limited to some specific cases, using Jensen's inequality and concavity of $\log$ det function, and apply the recent results obtained for static channels to the case of a stochastic (random) channel. We also demonstrate how to apply the results obtained for diversity combing to the MIMO system analysis using the upper bound above.

The upper bound derived in this paper accounts for both transmit and receive branch correlation in such a way that the impact of these branches can be estimated separately, which simplifies the computational procedure substantially

\section{MIMO CHANNEL CAPACITY}

For a fixed linear $n \times n$ matrix channel with additive white gaussian noise and when the transmitted signal vector is composed of statistically independent equal power components each with a gaussian distribution, the channel capacity is [3]:

$$
C=\log _{2} \operatorname{det}\left(\mathbf{I}+\frac{\rho}{n} \mathbf{H} \cdot \mathbf{H}^{+}\right) \mathrm{bits} / \mathrm{s} / \mathrm{Hz},
$$

where $n$ is the number of transmit/receive antennas (we consider here the case when the number of transmit and receive antennas are equal), $\rho$ is the signal-to-noise ratio (SNR), $\mathbf{I}$ is $\mathrm{n} \times \mathrm{n}$ identity matrix, $\mathbf{H}$ is the normalized 
channel matrix, which is considered to be frequency independent over the signal bandwidth, and "'" means transpose conjugate. We adopt here the following normalization condition:

$$
\sum_{i, j=1}^{n}\left|h_{i j}\right|^{2}=n,
$$

where $h_{i k}$ denotes the components of $\mathbf{H}$ ( $h_{i j}$ is the transfer factor between $j^{\text {th }}$ transmit antenna and $i^{\text {th }}$ receive antenna). Hence, $\rho / n$ is the average per-branch SNR, i.e. $\rho$ is the ratio of total received power (in all branches) to the per-branch noise level. Some other kinds of the normalization can also be used, but in this case $\rho / n$ will have a slightly different meaning.

\section{UPPER BOUND ON MIMO CHANNEL CAPACITY}

When the channel is random (stochastic), then the capacity is random, too. The mean (ergodic) capacity can be defined in this case as [1]:

$$
\langle C\rangle=\left\langle\log _{2} \operatorname{det}\left[\delta_{i j}+\frac{\rho}{n} \cdot r_{i j}\right]\right\rangle,
$$

where $r_{i j}$ is "instantaneous" correlation matrix,

$$
r_{i j}=\sum_{k} h_{i k} h_{j k} *
$$

$\delta_{i j}$ is Kroneker's delta, $<>$ is the expectation over the channel matrix. Note that Eq. (3) does take into account correlation occurring at both the transmit and receive ends. This equation can be used for statistical (MonteCarlo) simulations to evaluate $\langle C\rangle$ for some specific models of the channel matrix. However, these matrix numerical computations can be very lengthy, especially when the number of antennas is very large. Here we propose to use Jensen's inequality to dbtain an upper bound on $\langle C\rangle$. According to this inequality and concavity of $\log$ det function [8], one obtains:

$$
\langle C\rangle \leq \overline{C_{R}}=\log _{2} \operatorname{det}\left[\delta_{i j}+\frac{\rho}{n} \cdot r_{i j}^{R}\right]
$$

where $r_{i j}^{R}$ is the correlation matrix of receive branches,

$$
r_{i j}^{R}=\sum_{k}\left\langle h_{i k} h_{j k} *\right\rangle
$$

Note that this correlation matrix does not capture the correlation of transmit branches (since $k$ in (6) represents the transmit antenna index and it is the same for both factors). Thus, the upper limit in (5) can be close to the mean capacity when the correlation of receive branches is much higher than the correlation of transmit branches and, consequently, the effect of transmit branch correlation can be ignored. However, if the transmit correlation is higher than the receive one, then the upper bound in (5) is not an accurate approximation of the mean capacity. Therefore, in order to have an upper bound that is as close as possible to the mean capacity, one must also account for transmit correlation. To this end, the reciprocity of (1) can be used in the following way. First, we note that the MIMO capacity given by (1) is invariant under the transformation $\mathbf{H} \rightarrow \mathbf{H}^{\mathrm{T}}$ ("T" means transpose). This in effect is equivalent to reversing the direction of information transmission by interchanging transmit and receive ends. Thus, (3) still holds true if we define $r_{i j}$ as:

$$
r_{i j}=\sum_{k} h_{k i} h_{k j} *
$$

Hence, one obtains the second upper bound (the transmit bound),

$$
\langle C\rangle \leq \overline{C_{T}}=\log _{2} \operatorname{det}\left[\delta_{i j}+\frac{\rho}{n} \cdot r_{i j}^{T}\right]
$$

where $r_{i j}^{T}$ is the correlation matrix of transmit branches,

$$
r_{i j}^{T}=\sum_{k}\left\langle h_{k i} h_{k j} *\right\rangle,
$$

Note that the upper bound in (8) does not capture the receive correlation. Therefore, this upper bound will be close to the mean capacity when the transmit correlation is higher than the receive one. However, if the opposite is true, then this upper bound is not an accurate approximation of the mean capacity.

From inequalities (5) and (8) it is clear that a tighter upper bound of the mean channel capacity can be obtained by combining them. Thus, we form the compound upper bound by taking minimum of the two bounds defined above,

$$
\overline{C_{c m p}}=\min \left[\overline{C_{R}}, \overline{C_{T}}\right]
$$

This upper bound is much tighter than the receive or transmit bound considered separately when the transmit and receive branch correlations are significantly different.

Let us now consider an illustrative example of correlated Rayleigh channel. The components of $\mathbf{H}$ are taken to be identically distributed complex gaussian variables (real and imaginary parts are identically distributed and independent, i.e. the phase is uniformly distributed over $[0,2 \pi]$ ) with zero mean and unit variance. The correlation matrix of $\mathbf{H}$ is assumed to be of the following form: 


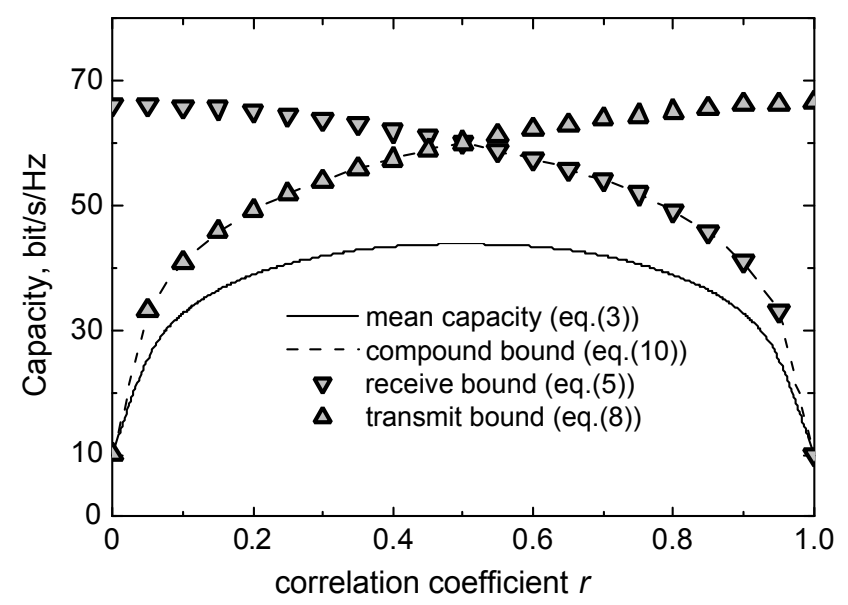

Figure 1. MIMO channel capacity and its upper bounds versus correlation coefficient

$$
R_{i j, k m}=\left\langle h_{i k} h_{j m} *\right\rangle=R_{i j}^{R} \cdot R_{k m}^{T},
$$

where $R_{i j}^{R}$ and $R_{i j}^{T}$ are uniform correlation matrixes of the receive and transmit branches correspondingly,

$$
R_{i j}^{R}=\left\{\begin{array}{ll}
r, & i \neq j \\
1, & i=j
\end{array}, \quad R_{k m}^{T}=\left\{\begin{array}{c}
1-r, \quad i \neq j \\
1, \quad i=j
\end{array},\right.\right.
$$

where $0 \leq r \leq 1$. In fact, (11) assumes that the receive and transmit branches are correlated independently on each other (which may be justified by the presence of local scatterers near both ends). Fig. 1 shows the mean capacity of this channel, obtained by extensive numerical simulations (Eq. 3), and the receive (Eq. 5), transmit (Eq. 8 ) and compound (Eq. 10) bounds. In this example, $r=0$ corresponds to uncorrelated receive branches and full correlation of the transmit ones; $r=1$ corresponds to full correlation of receive branches and uncorrelated transmit ones. The compound bound provides a good approximation to the mean capacity while the receive or transmit bounds alone are not accurate for the whole range of $r$. It is also interesting to note that the maximum capacity is achieved for $r=0.5$. This indicates that decrease in capacity is usually due to that side (transmit or receive) which has higher correlation. Thus, a rough estimation of the capacity may be obtained by considering only the higher correlated side.

\section{SOME ANALYTICAL RESULTS}

Using the upper bound on MIMO channel capacity derived above, one may apply the analytical results on the MIMO capacity of a deterministic channel $[5,6,10]$ to the case of random channel, i.e. to obtain the upper bound. For simplicity, we assume here that the transmit branches are not correlated and the correlation impact is due to the receive branch correlation. Obviously, the impact of transmit branch correlation can be estimated in a similar way and the combination of the results is trivial. It is also assumed that the receive power is identical for all the receive branches. In this case,

$$
\sum_{j}\left|h_{i j}\right|^{2}=1
$$

and $r_{i j}$ in (4) is the normalized correlation matrix, $\left|r_{i j}\right| \leq 1$. The effect of unequal received powers can be considered in a straightforward way [5].

We start with the uniform correlation matrix model, when all the correlation coefficients are equal and real [6],

$$
r_{i j}=\left\{\begin{array}{ll}
r, & i \neq j \\
1, & i=j
\end{array} \quad \operatorname{Im}[r]=0\right.
$$

This case is somewhat artificial because one expects that the correlation of neighbouring branches is larger than that of distant branches. However, the case of equal correlation coefficients provides a worst-case estimation and some insight into BLAST operation in correlated channels, so it deserves to be considered (besides, one may interpret $r$ as an "average" correlation coefficient). In this case, after some mathematical transformations that do not change the determinant, we present the upper bound (5) in the following form:

$$
\bar{C}=n \cdot \log _{2}\left(1+\frac{\rho}{n}(1-r)\right)+\log _{2}\left(1+\rho \cdot r \cdot\left(1+\frac{\rho}{n}(1-r)\right)^{-1}\right)
$$

For $n=2,(15)$ reduces to (8) in [5]. As a detail analysis of (7) shows, the channel capacity decreases substantially for $|r| \geq 0.5-0.8$, what agrees well with the recent measurements of the MIMO channel [9]. For $r=1,(15)$ reduces to the famous Shannon's formula. It should be noted that the second term in (15) is essential only when the correlation coefficient is close to 1 . However, in this case the advantage of the MIMO architecture over traditional techniques is very small (the channel capacity is close to the Shannon's SISO limit) and it is not reasonable to use it. Thus, when the MIMO architecture provides a substantial advantage, the upper bound on its channel capacity can be estimated as (for $0 \leq r<1$ ):

$$
\bar{C} \approx n \cdot \log _{2}\left(1+\frac{\rho}{n}(1-r)\right)
$$

In the limiting case of $n \rightarrow \infty$, one obtains from (16):

$$
\overline{C_{\infty}} \approx \frac{\rho(1-r)}{\ln 2}
$$

When $r=0$, the last two equations reduce to the wellknown formulas (in this case, $\mathbf{H}=\mathbf{I}$ ) [3]: 


$$
\bar{C}=n \cdot \log _{2}\left(1+\frac{\rho}{n}\right) \text { and } \overline{C_{\infty}}=\frac{\rho}{\ln 2}
$$

Comparison of (16) and (17) with (18) clearly indicates that the effect of the channel correlation is equivalent to the decrease in the SNR. Hence, for example, $r=0.5$ is equivalent to $3 \mathrm{~dB}$ reduction in SNR. Another interpretation of (16) and (17) is that the correlation of individual sub-channels gives an increase in the noise level because for each particular sub-channel all the other sub-channels are just the sources of interference.

Let us now consider the case of exponential correlation matrix [10],

$$
r_{i j}=\left\{\begin{array}{ll}
r^{j-i}, & i \leq j \\
r_{j i}^{*}, & i>j
\end{array}, \quad|r| \leq 1\right.
$$

Obviously, (19) may be not an accurate model for some real-world scenarios but this is a simple single-parameter model which allows one to study the effect of correlation on the MIMO capacity in an explicit way and to get some insight. It remains to be investigated whether this model is applicable or not to some specific scenarios. Note, however, that this model is physically reasonable in the sense that the correlation decreases with increasing distance between receive antennas. Thus, it should be more accurate than the uniform model above. In a practically-important case of high SNR $(\rho / n>>1), n>>1$ and $r<1$, (5) can be reduced to:

$$
\bar{C} \approx n \cdot \log _{2}\left(1+\frac{\rho}{n}\left(1-|r|^{2}\right)\right)
$$

In the limiting case of $n \rightarrow \infty$, one obtains from (20):

$$
\overline{C_{\infty}} \approx \frac{\rho}{\ln 2}\left(1-|r|^{2}\right)
$$

Comparison of (20) and (21) with (18) clearly indicates that the effect of the channel correlation is equivalent to the SNR loss, the same as for the uniform model above. Hence, for example, $r=0.7$ is equivalent to $3 \mathrm{~dB}$ reduction in the signal-to-noise ratio. Note also that the channel capacity does not depend on the correlation coefficient phase.

Fig. 2 shows the upper bound of MIMO channel capacity versus the correlation coefficient evaluated by the full matrix computation (Eqs. (5) and (19)) and by (20) for $n=10$ and 50, and $\rho=30 \mathrm{~dB}$. The MIMO channel capacity evaluated using the uniform correlation matrix model (see (15)) is also shown for comparison. As one may see from this figure, the accuracy of approximate formulas is quite good. It should be noted that the accuracy decreases as $n$ and $\rho / n$ decreases. The uniform model predicts lower capacity, as it should be (because it is the worst case model - the correlation between distant receive branches is the same as between neighboring

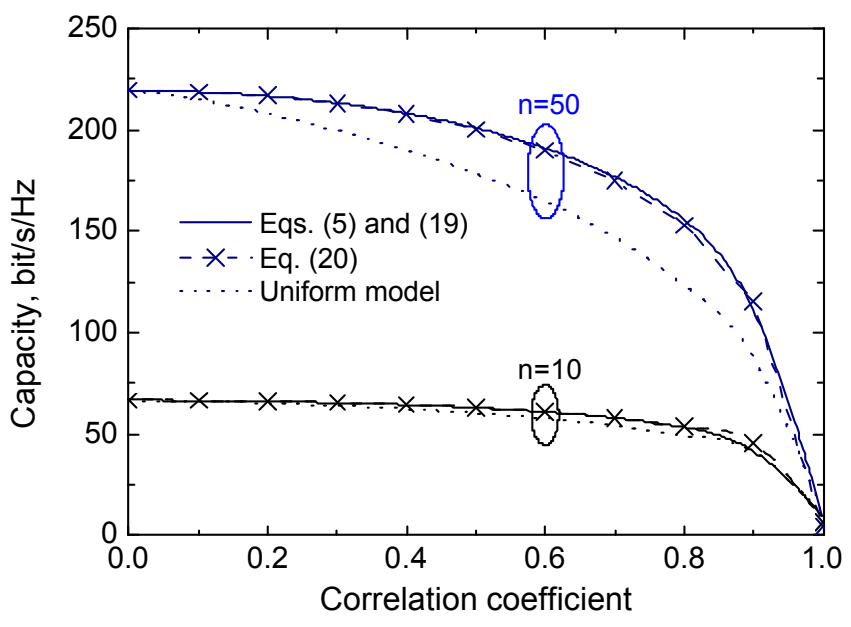

Figure 2. MIMO capacity upper bound versus correlation coefficient for exponential and uniform models.

ones). We also see that the MIMO capacity decreases significantly for $r>0.5-0.8$, that agrees well with the know results on the spatial diversity techniques [11] and with recent measurements of the MIMO channel [9]. Detailed analysis using Monte-Carlo simulations shows that the mean capacity is approximately 20 to $40 \%$ smaller then the upper bound above.

\section{PARADOX OF ZERO CORRELATION}

MIMO channel capacity is usually thought of as limited by correlation: it is low for highly correlated channel, and it is high when the correlation between individual subchannels (i.e. links between one transmit and one receive antenna) of the matrix (MIMO) channel is zero. However, an elegant example has been presented in [12], which demonstrates that zero correlation is not a guarantee of high capacity, i.e. the channel may have zero correlation and still only a single degree of freedom (these are so called degenerate channels or keyholes). However, no explanation has been provided to this phenomenon. In this section, we provide a statistical explanation of this phenomenon and, in particular, we emphasize that one should distinguish between "instantaneous" and "mean" (or conventional) correlation. We also present a general statistical criterion for the channel to be degenerate and propose a method to estimate the capacity of those channels.

Let us now consider $2 \times 2$ deterministic MIMO channel. (1) takes the following form in this case:

$$
C=\log _{2}\left(\left(1+\frac{\rho}{2}\right)^{2}-\left(\frac{\rho}{2}\right)^{2}\left|R_{12}\right|^{2}\right)+\log _{2}\left(r_{11} r_{22}\right)
$$


where $R_{12}$ is the normalized correlation coefficient $\left(\left|R_{12}\right| \leq 1\right)$,

$$
R_{12}=\frac{r_{12}}{\sqrt{r_{11} r_{22}}}
$$

In fact, $r_{11}$ and $r_{22}$ represent the normalized received power in $1^{\text {st }}$ and $2^{\text {nd }}$ branch correspondingly. Hence, the last term in (22) describes the effect of SNR. The eigenvalues $\lambda$ of $r_{i j}$ can be obtained from the following equation:

$$
\lambda^{2}-\left(r_{11}+r_{22}\right) \lambda+r_{11} r_{22}\left(1-\left|R_{12}\right|^{2}\right)=0
$$

The singular values of $h_{i j}$ are square roots of $\lambda$ [1]. Thus, correlation has the major impact on the number of degrees of freedom (i.e., non-zero singular or eigenvalues): there are two degrees of freedom when $\left|R_{12}\right|<1$ and only one when $\left|R_{12}\right|=1$, as long as the received powers are not zero.

When the channel is random, the mean (ergodic) capacity may be defined using the expectation over the channel matrix in (22) (see eq. (3)) [1]. Detailed analysis using Monte-Carlo simulations of the correlated Rayleigh channel and of the channel in [12] shows that the impact of the second term in (22) on the mean capacity is much smaller than that of the first term for $\rho>>1$. The same conclusion may be obtained using Jensen's inequality. The second term mainly accounts for varied received powers. On the contrary, correlation has the major impact on the mean capacity. Hence, to isolate and study the effect of correlation, we neglect the second term. In this case, the mean capacity depends on $R_{12}$ only, which is "instantaneous" correlation coefficient, and can be presented as follows:

$$
\langle C\rangle=\int_{D R_{12}} C\left(R_{12}\right) \cdot f\left(R_{12}\right) d R_{12}
$$

where $f\left(R_{12}\right)$ is the probability density function (PDF) of $R_{12}$, and $D R_{12}$ is the range of $R_{12}$. Thus, the mean capacity depends on the PDF of $R_{12}$, not only on its mean value. In general, the mean correlation is not a reliable tool in estimating the MIMO capacity of a random channel.

Let us now consider an illustrative example, when $R_{12}= \pm 1$ with equal probability. Obviously, the mean correlation is zero but the mean capacity is low and there is only one degree of freedom just because $\left|R_{12}\right|=1$. The next example is provided in [12]. In that case,

$$
R_{12}=e^{j\left(\varphi_{1}-\varphi_{2}\right)}
$$

where $\varphi_{1}=\arg \left(b_{1}\right), \quad \varphi_{2}=\arg \left(b_{2}\right)$, and $b_{1}$ and $b_{2}$ are scattering coefficients (see [12] for detail discussion). Again, $\left\langle R_{12}\right\rangle=0$ because $\varphi_{1}$ and $\varphi_{2}$ are independent and uniformly distributed, but the mean capacity is low and there is only one degree of freedom because $\left|R_{12}\right|=1$. From the considerations above, we may conclude the following:

- The capacity of deterministic channel is maximum when $R_{12}=0$ (see eq. (22)). However, as the examples above show, it is wrong to state the same about random channel using the mean correlation, i.e. zero mean correlation of a random channel is not a sufficient condition of maximum mean capacity. Using inequality (5), we conclude that it is the necessary condition (i.e., if the mean correlation is high, than the mean capacity is necessarily low).

- Referring to eq. (22), we conclude that the sufficient condition of high capacity is low mean magnitude correlation. For example, if $\left\langle\left|R_{12}\right|\right\rangle=0$, then there are two degrees of freedom and the mean capacity is maximum simply because $R_{12}=0$ in this case.

The general conditions for a channel to be degenerate are,

$$
\left\langle R_{12}\right\rangle=0 \text { and }\left|R_{12}\right|=1
$$

From practical viewpoint, $\left|R_{12}\right|$ may not be equal to 1 but be close to it. The capacity will be low in this case as well. In particular, according to the results of Section 4, it will be low when $\left|R_{12}\right| \geq 0.5-0.8$. In the case of degenerate channels, the mean correlation does not provide an accurate estimation of the capacity. Figure 3 illustrates the channel capacity for PDF of $R_{12}$ of the following form:

$$
f\left(R_{12}\right)=c \cdot \exp \left(\frac{\left|R_{12}\right|}{\alpha}\right), \quad\left|R_{12}\right| \leq 1
$$

where $c$ is normalizing constant, and $\alpha$ determines the root-mean-square (RMS) value of $R_{12}$ (note that $\alpha$ may be negative as well as positive). Obviously, the mean correlation is zero and its use for estimating the capacity will give an incorrect result. As figure 3 indicates, a more accurate estimation of the capacity of degenerate channels can be obtained using RMS or mean magnitude correlation.

It is interesting to note that the eigenvalue approach, which is widely used for the MIMO system analysis, is more formal mathematically and does not provide this insight. 


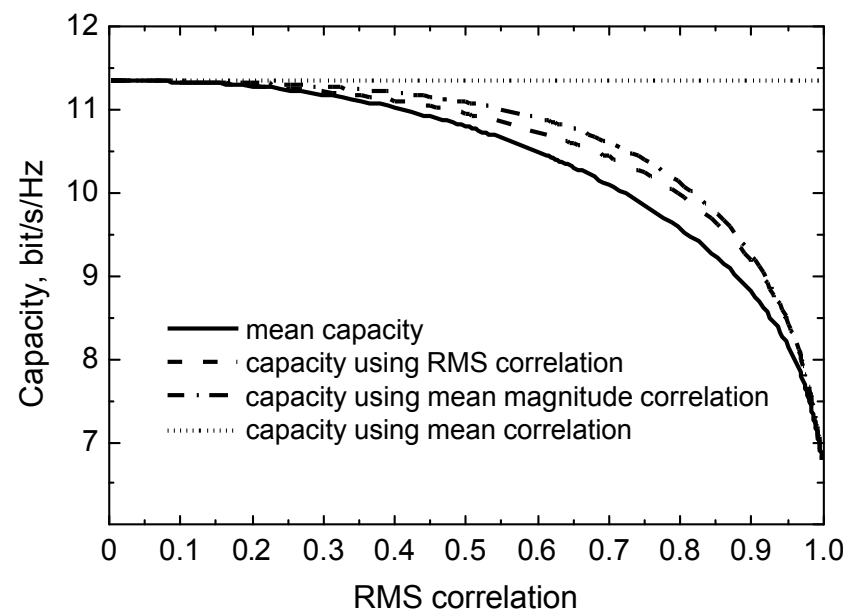

Figure 3. MIMO capacity using RMS, mean magnitude and mean correlation versus RMS correlation for $\rho=20 \mathrm{~dB}$.

\section{CONCLUSIONS}

In this paper, we discussed the use of Jensen inequality to estimate the MIMO channel capacity through the upper bound on it. The direct application of the inequality results in the receive branch upper bound, which does not account for the transmit branch correlation. The transmit branch bound is obtain using the transpose of the channel matrix. Finally, the compound bound is formed using the minimum of the two bounds above. This compound bound accounts for both transmit and receive branch correlation in such a way that the impact of these branches can be estimated separately, which simplifies the procedure substantially. Extensive numerical simulations confirm that this bound is a quite accurate estimation of the mean MIMO capacity. The statistics of amplitude distribution of the matrix channel coefficients has no significant impact on the capacity - the main impact is due to correlation.

Using the bound above, we applied the results on MIMO capacity of deterministic channels to a random channel, i.e. estimated the capacity upper bound using the uniform and exponential correlation matrix models. These estimations agree well with the recent measurements of the MIMO radio channel. We have also shown that the increase in correlation is equivalent to the decrease in SNR.

Finally, we have discussed the paradox of zero correlation and provided a statistical explanation for it. In particular, we have shown that one should distinguish between "average" (conventional) and "instantaneous" correlation. High magnitude correlation is the solution to this paradox. Zero average correlation is not a guarantee of high capacity. On the contrary, zero or low mean magnitude correlation is indeed a guarantee of high capacity. Mean magnitude or RMS correlation should be used for the capacity estimation of degenerate channels.

\section{REFERENCES}

[1] I.E. Telatar, "Capacity of Multi-Antenna Gaussian Channels," AT\&T Bell Lab. Internal Tech. Memo., June 1995.

[2] G.J Foschini, 'Layered space-time architecture for wireless communication in a fading environment when using multiple antennas', Bell Lab. Tech. J., vol. 1, N. 2, pp. 41-59, 1996.

[3] Foschini, G.J., Gans M.J.: 'On Limits of Wireless Communications in a Fading Environment when Using Multiple Antennas', Wireless Personal Communications, vol. 6, No. 3, pp. 311-335, March 1998.

[4] G.D. Golden, G.J. Foschini, R.A. Valenzuela, P.W. Wolniansky, 'Detection Algorithm and Initial Laboratory Results Using V-BLAST Space-Time Communication Architecture', Electronics Letters, vol. 35, No. 1, pp.14-16, $7^{\text {th }}$ January 1999.

[5] S.L. Loyka, "Channel Capacity of Two-Antenna BLAST Architecture," Electronics Letters, vol. 35, No. 17, pp. 1421-1422, 19th Aug. 1999.

[6] S.L. Loyka, J.R Mosig, 'Channel Capacity of NAntenna BLAST Architecture,' Electronics Letters, vol. 36, No.7, pp. 660-661, Mar. 2000.

[7] Shiu, D.S., Foschini, G.J., Gans, M.J., Kahn, J.M., 'Fading Correlation and Its Effect on the Capacity of Multielement Antenna Systems,' IEEE Trans. on Communications, v. 48, N. 3, Mar. 2000, pp. 502513.

[8] T.M. Cover, J.A. Thomas, Elements of Information Theory, John Wiley \& Sons, New York, 1991

[9] C.C. Martin, J.H. Winters, N.S. Sollenberger, Multiple-Input Multiple-Output (MIMO) Radio Channel Measurements, IEEE VTC'2000 Fall Conference, Sept. 24-28 2000, Boston, USA.

[10] S.L. Loyka, Channel Capacity of MIMO Architecture Using the Exponential Correlation Matrix, IEEE Communication Letters, 2000, submitted for publication

[11] Jakes, W.C. Jr.: 'Microwave Mobile Communications', John Wiley and Sons, New York, 1974.

[12] D. Chizhik, G.J. Foschini, R.A. Valenzuela, 'Capacities of multi-element transmit and receive antennas: Correlations and keyholes', Electronics Letters, vol. 36, No. 13, pp.1099-1100, $22^{\text {nd }}$ June 2000 